\title{
Novel Spin-Orbital Phases Induced by Orbital Dilution
}

\author{
Wojciech Brzezicki ${ }^{1,2}$ - Mario Cuoco ${ }^{1,2}$ - Andrzej M. Oleś ${ }^{3,4}$
}

Received: 10 October 2015 / Accepted: 19 November 2015 / Published online: 22 December 2015

(C) The Author(s) 2015. This article is published with open access at Springerlink.com

\begin{abstract}
We demonstrate that magnetic $3 d$ impurities with $S=3 / 2$ spins and no orbital degree of freedom induce changes of spin-orbital order in a $4 d^{4}$ Mott insulator with $S=1$ spins. Impurities act either as spin defects which decouple from the surrounding ions, or trigger orbital polarons along $3 d-4 d$ bonds. The $4 d-4 d$ superexchange in the host $J_{\text {host }}$ competes with $3 d-4 d$ superexchange $J_{\text {imp }}$-it depends on which orbital is doubly occupied. The spinorbital order within the host is totally modified at doping $x=1 / 4$. Our findings provide new perspective for future theoretical and experimental studies of doped transitionmetal oxides.
\end{abstract}

Keywords Orbital dilution · Spin-orbital order · Orbital polarons $\cdot$ Doped Mott insulator

\section{Orbital Dilution}

Entangled spin-orbital superexchange interactions lead to several surprises in transition-metal oxides [1]. In Mott

Andrzej M. Oleś

a.m.oles@fkf.mpg.de

1 CNR-SPIN, IT-84084 Fisciano, SA, Italy

2 Dipartimento di Fisica "E. R. Caianiello", Universitá di Salerno, IT-84084 Fisciano, SA, Italy

3 Marian Smoluchowski Institute of Physics, Jagiellonian University, Prof. S. Łojasiewicza 11, PL-30348 Kraków, Poland

4 Max Planck Institute for Solid State Research, Heisenbergstrasse 1, D-70569 Stuttgart, Germany insulators, these interactions are modified by doping which may generate novel phases-they emerge from the interplay of complex spin-orbital-charge couplings. For instance, hole doping of a ferromagnetic (FM) system with $d^{1}$ ionic configurations removes locally orbital degrees of freedom and generates stripe phases with orbital polarons [2]. We show below that similar polarons emerge also in doped spin-orbital systems.

The well-known example are hole-doped $\mathrm{La}_{1-x} \mathrm{Sr}_{x} \mathrm{MnO}_{3}$ manganites with colossal magnetoresistance [3]. At low doping, orbital polarons emerge in an antiferromagnetic (AF) system by double-exchange mechanism [4], while at higher doping, spin order changes globally to a FM metal coexisting with an $e_{g}$ orbital liquid phase [5]. But a different scenario is also possible-frustrated spin-orbital interactions may lead in some cases to the collapse of any long range order and to a spin-orbital liquid suggested for $\mathrm{LiNiO}_{2}$ [6]. However, spin and orbital energy scales are here quite different, and the reasons behind the absence of magnetic long range order are indeed more subtle and not yet fully understood [7].

A rather unique example of a spin-orbital system are perovskite vanadates, where interesting competition between two types of spin-orbital order was observed [8]. The theoretical spin-orbital model describes an interplay between $S=1$ spins and $\tau=1 / 2$ orbital doublet $\{y z, z x\}$ active along the $c$ axis [9], while $x y$ orbitals are filled by one electron each. In addition, $G$-type $\mathrm{AF}(G-\mathrm{AF})$ order in $\mathrm{YVO}_{3}$ is fragile and switches to $C$-type $\mathrm{AF}(C-\mathrm{AF})$ one in $\mathrm{Y}_{1-x} \mathrm{Ca}_{x} \mathrm{VO}_{3}$ [10], with staggered lines of one-dimensional (1D) FM order (Fig. 1), accompanied by $G$-type alternating orbital $(G-\mathrm{AO})$ order. Already at low doping, $x \simeq 0.02$ finite spectral weight is generated within the Mott-Hubbard gap due to charge defects away from the $\mathrm{VO}_{6}$ octahedra [11]. 


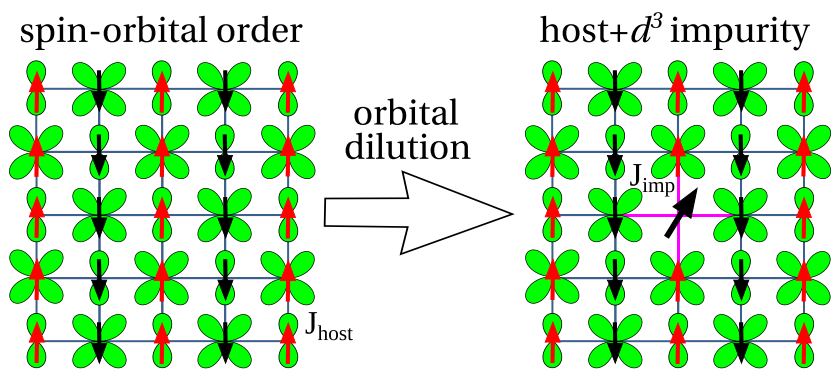

Fig. 1 Left-spin-orbital $C-\mathrm{AF} / G-\mathrm{AO}$ order found in Mott insulators with $d^{2}$ (vanadates) or $d 4$ (ruthenates) ionic configurations. Spins $S=1$ are shown by red (black) arrows and doubly occupied $t_{2 g}$ orbitals (doublons) are shown by green symbols for $a$ and $c$ orbitals. Right - the orbital dilution occurs after doping by a $3 d^{3}$ ion with $S=3 / 2$ and no orbital degree of freedom. Here, $a$ doublon site is replaced by $\mathrm{Mn}^{4+}$ or $\mathrm{Cr}^{3+}$ impurity coupled to the host by $3 d-4 d$ bonds

Here, we consider doping by static charge defects, e.g., $\mathrm{Mn}^{4+}$ or $\mathrm{Cr}^{3+}$ ions, within the $a b$ planes having columnar $C$-AF order of $S=1$ spins at ruthenium or vanadium ions in the host which leads to orbital dilution [12] (see Fig. 1). In contrast to hole-doped manganites, holes are here immobile and disturb $t_{2 g}$ orbital order. It has been shown that (i) dilute $\mathrm{Cr}$ doping for $\mathrm{Ru}$ reduces the temperature of the orthorhombic distortion and induces $\mathrm{FM}$ behavior in $\mathrm{Ca}_{2} \mathrm{Ru}_{1-x} \mathrm{Cr}_{x} \mathrm{O}_{4}$ (with $0<x<0.13$ ) [13] and (ii) Mn-substituted single crystals of $\mathrm{Sr}_{3} \mathrm{Ru}_{2-x} \mathrm{Mn}_{x} \mathrm{O}_{7}$ reveal an unusual $E$-type AF structure at $x=0.16$ [14] which is again triggered by double exchange. These findings motivate the theoretical search for the consequences of orbital dilution.

\section{Isolated Impurities}

For $\mathrm{Ca}_{2} \mathrm{RuO}_{4}$ host, we use the spin-orbital model derived for $t_{2 g}^{4}$ ions with low $(S=1)$ spins [15]. This model uses $t_{2 g}$ doublons as orbital degrees of freedom and is isomorphic to the vanadate $d^{2}$ model [9], with the doublons transforming into empty orbitals (occupied by two holes). We shall label $t_{2 g}$ orbitals by index $\gamma$ when a given orbital is inactive along a direction $\gamma \in\{a, b, c\}$ :

$|a\rangle \equiv|y z\rangle, \quad|b\rangle \equiv|x z\rangle, \quad|c\rangle \equiv|x y\rangle$.

We consider a two-dimensional (2D) square lattice with transition-metal ions connected via oxygen orbitals as in an $a b \mathrm{RuO}_{2}$ plane of $\mathrm{Ca}_{2} \mathrm{RuO}_{4}\left(\mathrm{SrRuO}_{3}\right)$. In this case, $|a\rangle(|b\rangle)$ orbitals are active along the $b(a)$ axis, while $|c\rangle$ orbitals are active along both axes, $a$ and $b$. The superexchange in the host for the bonds $\langle i j\rangle$ along the $\gamma \in\{a, b\}$ axis,

$\mathcal{H}_{4 d-4 d}=J_{\text {host }} \sum_{\langle i j\rangle \| \gamma}\left\{J_{i j}^{(\gamma)}\left(\mathbf{S}_{i} \cdot \mathbf{S}_{j}+1\right) J_{i j}+K_{i j}^{(\gamma)}\right\}$, is given by $J_{\text {host }}$ and depends on orbital operators, $J_{i j}^{(\gamma)}$ and $K_{i j}^{(\gamma)}$ [15]. The above form is generic [1], and the interactions depend on the intraorbital Coulomb $U_{2}$ element and Hund's exchange $J_{2}$ in the host.

We introduce two parameters to characterize the interactions along the impurity-host $3 d-4 d$ bonds [12]:

$J_{\text {imp }}=\frac{t^{2}}{4 \Delta}, \quad \eta_{\text {imp }}=\frac{J_{1}}{\Delta}$.

Here, the charge excitations $3 d_{i}^{3} 4 d_{j}^{4} \Rightarrow 3 d_{i}^{4} 4 d_{j}^{3}$ determine the $d^{3}-d^{4}$ superexchange [12] and involve the energy

$\Delta=I_{e}+3\left(U_{1}-U_{2}\right)-4\left(J_{1}-J_{2}\right)$.

It depends on the onsite Coulomb interactions $\left\{U_{m}\right\}$ ( $m=1$ stands for the impurity $d^{3}$ ion), on Hund's exchange $\left\{J_{m}\right\}$, and on the ionic energy $I_{e}$. For $\mathrm{Mn}$ or $\mathrm{Cr}$ impurities in ruthenates, $\Delta>0$.

With the parametrization introduced above, the dominant term in the impurity-host Hamiltonian for the impurity spin $\mathbf{S}_{i}$ interacting with the neighboring host spins $\left\{\mathbf{S}_{j}\right\}$ for nearest neighbors $j \in \mathcal{N}(i)$ can be written in a rather compact form as follows:

$\mathcal{H}_{3 d-4 d}(i) \simeq \sum_{\gamma, j \in \mathcal{N}(i)}\left\{J_{S}\left(D_{j}^{(\gamma)}\right)\left(\mathbf{S}_{i} \cdot \mathbf{S}_{j}\right)+E_{D} D_{j}^{(\gamma)}\right\}$,

where the spin couplings $J_{S}\left(D_{j}^{(\gamma)}\right)$ depend on orbital (doublon) configuration, $D_{j}^{(\gamma)}$ is the doublon projection operator at site $j$ and the doublon energy $E_{\mathrm{D}}$ depends on $\eta_{\text {imp }}$ (3). It can be shown [12] that the dominant energy scale is $E_{\mathrm{D}}^{\gamma}$, so for a single $3 d-4 d$ bond, the doublon does not occupy the inactive $(\gamma)$ orbital and spins couple with $J_{S}\left(D_{j}^{(\gamma)}=0\right)$ which can be either AF if $\eta_{\text {imp }}<0.43$ or FM if $\eta_{\text {imp }}>0.43$. The change of sign at $\eta_{\mathrm{imp}}^{c} \simeq 0.43$ is reminiscent of that found in the Kugel-Khomskii model and as there [16] could lead to exotic spin phases.

It has been found that a single $d^{3}$ impurity at site $i$ modifies the spin-orbital order at its nearest neighbors $j \in \mathcal{N}(i)$, while second nearest neighbors were assumed [12] to follow the $C-\mathrm{AF} / G-\mathrm{AO}$ order in the host (Fig. 1). Here, we release this constraint and consider classically the impurity with its first, second, and third nearest neighbors. One finds that the phase diagram is almost unchanged by increasing cluster size from [12] for the doping at $c$ doublon and doublon orbitals at nearest neighbor sites change from inactive to active ones with increasing $J_{\text {imp }}$ (not shown), the latter similar to orbital polarons in manganites [17].

In contrast, for $a$ doublon doping, the impurity flips $a$ to $b$ orbitals at second neighbors along the vertical line and reverses four spins along the $b$ axis (replacing a 1D FM order along if) in phase $\mathrm{AF} a 1$ for small $\eta_{\text {imp }}$ (see Fig. 2). This shows that the modification of spin-orbital 


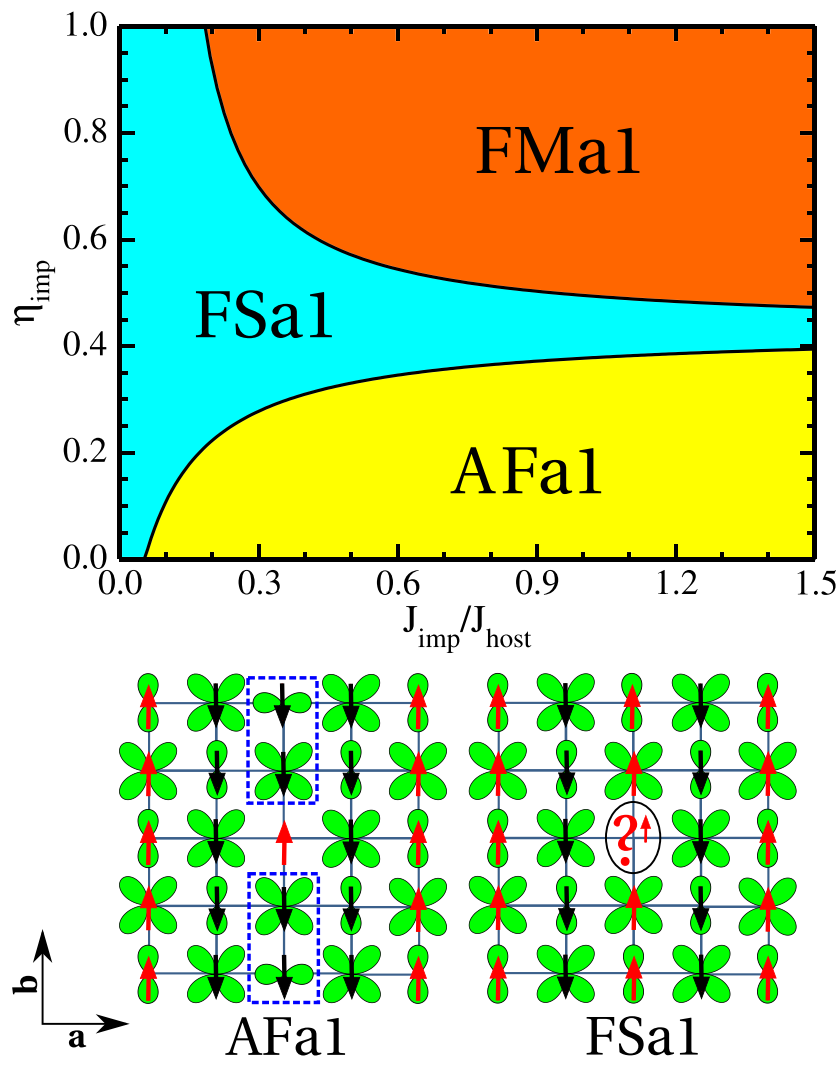

Fig. 2 Phase diagram for the $3 d$ impurity in the $a b$ plane of $4 d$ host with $C$-AF/ $G$-AO order and impurity replacing $a$ orbital doublon. Spin order is switched on the central vertical line in phase $\mathrm{AF} a 1$ (dashed boxes), while in $\mathrm{FS} a 1$ phase, the impurity spin is frustrated and unable to alter $C$-AF pattern in the host; at large $\eta_{\text {imp }}$, FM order is found in FM $a 1$ phase

order induced by $3 d-4 d$ bonds may be long range. Surprisingly, the host $C-\mathrm{AF} / G-\mathrm{AO}$ order recovers for larger $\eta_{\text {imp }} \simeq \eta_{\text {imp }}^{c}$ in frustrated spin $\mathrm{FS} a 1$ phase when the AF $3 d-4 d$ coupling weakens. Here, the quantum fluctuations support the impurity spin following $C$-AF spin order. Finally, when the impurity-host superexchange becomes strongly $\mathrm{FM}$, the impurity spin in $\mathrm{AF} a 1$ phase flips and FM $a 1$ phase emerges.

\section{Phase Diagram at $x=1 / 4$ Doping}

As for a single impurity, the $3 d-4 d$ bonds strongly influence spin-orbital order at finite doping except for a relatively narrow window of Hund's exchange $\eta_{\mathrm{imp}} \simeq \eta_{\mathrm{imp}}^{c}$. Indeed, already for intermediate doping, $x=1 / 9,1 / 8$ or $1 / 5$, spinorbital order may change globally [12]. Here, we investigate higher periodic doping $x=1 / 4$, where half of the superexchange bonds are $3 d-4 d$ hybrid ones, and we show that they dominate and dictate the overall spin-orbital order.
One finds that when at least one of the two impurity parameters is small, either $J_{\text {imp }}$ or $\eta_{\text {imp }}$ (3), $c$ doublon sites are doped and host-impurity coupling is AF. This AF coupling is amplified by charge excitations on $3 d-4 d$ bonds when doublon orbitals at $4 d$ ions are inactive-it dominates in this range of the phase diagram and induces FM order along the $4 d-4 d$ host bonds within the antiferrimagnetic (AFI) spin order for the entire $a b$ plane, see Fig. 3. Thus only every second vertical line is FM, as in the initial $C$-AF phase, while host spins are inverted on any other vertical line and the doublon orbitals flip from $a$ to $b$. Indeed, this modification of the orbital order stabilizes the FM interactions for $c-a$ doublon pairs on the horizontal bonds. For FM order, this orbital pattern is stabilized by double exchange, similar to a hole doped $t_{2 g}$ system [2]. In contrast, if either $J_{\text {imp }} / J_{\text {host }}$ or $\eta_{\text {imp }}$ is bigger than $\sim 0.5$, orbital dilution occurs on sublattice $a$. When the $3 d-4 d$ superexchange is $\mathrm{AF}$, the orbital order along the undoped vertical FM lines
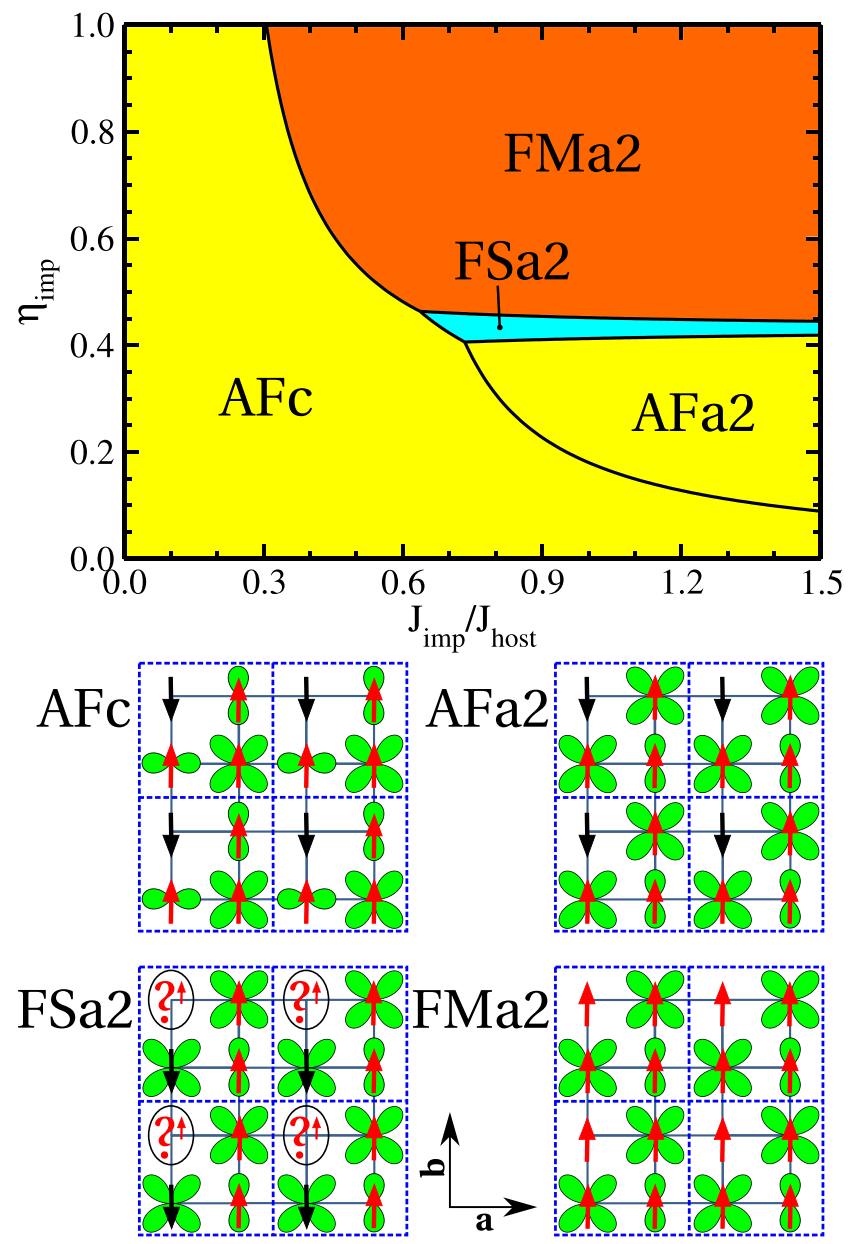

Fig. 3 Ground state diagram obtained for periodic $x=1 / 4$ doping of $d^{4}$ spin-orbital host by $d^{3}$ ions (arrows). Dashed blue lines separate $2 \times 2$ unit cells in different phases; question marks in FSa2 indicate classically frustrated spins which order (small arrows) due to quantum fluctuations 
is unchanged and one finds in phase $\mathrm{AF} a 2$ again the same spin order as in $\mathrm{AF} c$, but now there are twice as many $c$ doublons as $a$ ones.

As in the dilute limit (Fig. 2), impurity spins are frustrated also at $x=1 / 4$ doping in phase $\mathrm{FS} a 2$ when $\eta_{\text {imp }} \simeq \eta_{\text {imp }}^{c}$ (Fig. 3) and the $3 d-4 d$ superexchange nearly vanishes and changes sign. Then, the host spins in between experience almost entirely the AF $4 d-4 d$ superexchange and follow $C$-AF order. Quantum fluctuations stabilize opposite to them orientation of impurity spins, and this phase may be seen as a precursor of FMa2 phase which has again $75 \%$ FM bonds as the two AFI phases. At sufficiently large $\eta_{\text {imp }}$, FM order takes over in FMa 2 phase (except for the range of $c$ doublon doping). The $G-\mathrm{AO}$ order is the same in this latter phase as in the undoped host (see Fig. 3).

\section{Discussion and Summary}

Orbital dilution will play a role in several Mott insulators with spin-orbital order doped by ions without active orbitals. We have shown that the orbital order around impurities changes in general, so even in the dilute limit one may expect observable effects due to islands of reversed spins and doublon orbitals.

The phase diagram of Fig. 3 confirms the general rule that only a sufficiently strong coupling $J_{\text {imp }} / J_{\text {host }}$ leads to orbital dilution on $a$ sublattice and to a rich competition between various types of spin order [12]. We argue that the general trends found here are generic and the phase diagrams are only quantitatively modified by quantum fluctuations, at least in systems with weak spin-orbit coupling. We have found that double exchange leads to local or global changes of spin-orbital order, similar to formation of orbital stripes [2] or orbital polarons in doped manganites [4]. Such changes are expected to generate novel spinorbital-charge modulated patterns reported recently for $t_{2 g}$ systems [18].

In summary, this study highlights the role played by orbital dilution due to $d^{3}$ impurities in cubic spin-orbital systems and opens a new avenue towards theoretical understanding of Mn-doped layered ruthenates and related systems. We have shown that impurities change radically the spin-orbital order around them in the entire parameter range. As a general feature, one finds frustrated impurity spins and their ability to polarize the host orbitals around them. This property is remarkable and concerns both the dilute and high doping regime, so one expects global changes of spin-orbital order in doped materials. It is challenging to investigate whether disordered impurities would generate similar changes of spin-orbital order as well.
Acknowledgments Open access funding provided by Max Planck Society. W.B. acknowledges support by the European Unions Horizon 2020 research and innovation program under the Marie SklodowskaCurie Grant Agreement No. 655515. M.C. acknowledges funding by the European Union FP7/2007-2013 program, Grant Agreement No. 264098-MAMA. We acknowledge support by Narodowe Centrum Nauki (NCN, National Science Center), Project No. 2012/04/A/ST3/00331.

Open Access This article is distributed under the terms of the Creative Commons Attribution 4.0 International License (http:// creativecommons.org/licenses/by/4.0/), which permits unrestricted use, distribution, and reproduction in any medium, provided you give appropriate credit to the original author(s) and the source, provide a link to the Creative Commons license, and indicate if changes were made.

\section{References}

1. Oleś, A.M.: Fingerprints of spin-orbital entanglement in transition metal oxides. J. Phys.: Condens. Matter 24, 313201 (2012)

2. Wróbel, P., Oleś, A.M.: Ferro-orbitally ordered stripes in systems with alternating orbital order. Phys. Rev. Lett. 104, 206401 (2010)

3. Tokura, Y.: Critical features of colossal magnetoresistive manganites. Rep. Prog. Phys. 69, 797 (2006)

4. Kilian, E., Khaliullin, G.: Orbital polarons in the metal-insulator transition of manganites. Phys. Rev. B 60, 13458 (1999)

5. Feiner, L.F., Oleś, A.M.: Orbital liquid in ferromagnetic manganites: the orbital Hubbard model for $e_{g}$ electrons. Phys. Rev. B 71, $144422(2005)$

6. Vernay, F., Penc, K., Fazekas, P., Mila, F.: Orbital degeneracy as a source of frustration in $\mathrm{LiNiO}_{2}$. Phys. Rev. B 70, 014428 (2004)

7. Reitsma, A., Feiner, L.F., Oleś, A.M.: Orbital and spin physics in $\mathrm{LiNiO}_{2}$ and $\mathrm{NaNiO}_{2}$. New J. Phys. 7, 121 (2005)

8. Fujioka, J., Yasue, T., Miyasaka, S., Yamasaki, Y., Arima, T., Sagayama, H., Inami, T., Ishii, K., Tokura, Y.: Critical competition between two distinct orbital-spin ordered states in perovskite vanadates. Phys. Rev. B 82, 144425 (2010)

9. Khaliullin, G., Horsch, P., Oleś, A.M.: Theory of optical spectral weights in Mott insulators with orbital degrees of freedom. Phys. Rev. B 70, 195103 (2004)

10. Fujioka, J., Miyasaka, S., Tokura, Y.: Doping variation of anisotropic charge and orbital dynamics in $\mathrm{Y}_{1-x} \mathrm{Ca}_{x} \mathrm{VO}_{3}$ : comparison with $\mathrm{La}_{1-x} \mathrm{Sr}_{x} \mathrm{VO}_{3}$. Phys. Rev. B 77, 144402 (2008)

11. Avella, A., Oleś, A.M., Horsch, P.: Defects, disorder, and strong electron correlations in orbital degenerate, doped Mott insulators. Phys. Rev. Lett. 115, 206403 (2015)

12. Brzezicki, W., Oleś, A.M., Cuoco, M.: Spin-orbital order modified by orbital dilution in transition-metal oxides: from spin defects to frustrated spins polarizing host orbitals. Phys. Rev. X 5, 011037 (2015)

13. Qi, T.F., Korneta, O.B., Parkin, S., De Long, L.E., Schlottmann, P., Cao, G.: Negative volume thermal expansion via orbital and magnetic orders in $\mathrm{Ca}_{2} \mathrm{Ru}_{1-x} \mathrm{Cr}_{x} \mathrm{O}_{4}(0<x<0.13)$. Phys. Rev. Lett. 105, 177203 (2010)

14. Mesa, D., Ye, F., Chi, S., Fernandez-Baca, J.A., Tian, W., Hu, B., Jin, R., Plummer, E.W., Zhang, J.: Single-bilayer $E$-type antiferromagnetism in $\mathrm{Mn}$-substituted $\mathrm{Sr}_{3} \mathrm{Ru}_{2} \mathrm{O}_{7}$ : neutron scattering study. Phys. Rev. B 85, 180410(R) (2012) 
15. Cuoco, M., Forte, F., Noce, C.: Interplay of Coulomb interactions and $c$-axis octahedra distortions in single-layer ruthenates. Phys. Rev. B 74, 195124 (2006)

16. Brzezicki, W., Dziarmaga, J., Oleś, A.M.: Noncollinear magnetic order stabilized by entangled spin-orbital fluctuations. Phys. Rev. Lett. 109, 237201 (2012)
17. Daghofer, M., Oleś, A.M., von der Linden, W.: Orbital polarons versus itinerant $e_{g}$ electrons in doped manganites. Phys. Rev. B 70, 184430 (2004)

18. Brzezicki, W., Noce, C., Romano, A., Cuoco, M.: Zigzag and checkerboard magnetic patterns in orbitally directional doubleexchange systems. Phys. Rev. Lett. 114, 247002 (2015) 